\title{
Determination of the serine palmitoyl transferase inhibitor Myriocin by electrospray and Q-trap mass spectrometry
}

\author{
Giuseppe Matteo Campisi ${ }^{\text {a }}$, Paola Signorelli ${ }^{\text {a }}$, Jessica Rizzo ${ }^{\text {b }}$, Claudio Ghilardi ${ }^{\text {b }}$, \\ Jacopo Antognetti ${ }^{\text {b }}$, Anna Caretti a , Jelena S. Lazarevic' ${ }^{\text {c }}$, Enrica Strettoi d, Elena
} Novelli $^{\text {e }}$, Riccardo Ghidoni ${ }^{\text {a }}$, Federico Maria Rubino ${ }^{\text {f }}$, Rita Paroni ${ }^{\text {b }}$

${ }^{\mathrm{a}}$ Laboratory of Biochemistry and Molecular Biology, ${ }^{\mathrm{b}}$ Laboratory of Analytical Chemistry and Clinical Biochemistry, ${ }^{\mathrm{f}}$ Laboratory for Analytical Toxicology and Metabolomics (LaTMA) and International Centre for Rural Health, Department of Health Science, University of Milan, Milan, Italy.

${ }^{\mathrm{d}}$ Neuroscience Institute, Consiglio Nazionale delle Ricerche, Pisa 56100, Italy;

${ }^{\mathrm{e}}$ G.B. Bietti Foundation for Ophthalmology, Rome 00198, Italy

${ }^{c}$ Department of Chemistry, Faculty of Medical Sciences, University of Niš , Bul. Dr. Zorana Dindică 81, RS-18000 Nis (Serbia).

\section{Correspondence}

Rita Paroni Associate Professor in Clinical Biochemistry Dpt. of Health Science (DISS), University of Milan, via di Rudinì 8, 20142 Milano Office +39-02-50323272 - Fax +39-0250323274 email: rita.paroni@unimi.it

Running Title: Myriocin in biological matrix by LC-MS

Abbreviations: Myriocin: Myr; fluorenylmethylchlorofomate: FMOC; 14-HydroxyMyriocin: 14-OH-Myr; Myr loaded-solid lipid nanoparticles: SLN-Myr; working solution: WS; Limit-of-Detection: LoD; Lower-Limit-of-Quantification: LLoQ

This article has been accepted for publication and undergone full peer review but has not been through the copyediting, typesetting, pagination and proofreading process which may lead to differences between this version and the Version of Record. Please cite this article as doi: 


\begin{abstract}
Myriocin, is a potent inhibitor of serine-palmitoyl-transferase, the first and rate-determining enzyme in the sphingolipids biosynthetic pathway. This study developed, validated and applied a LC-MS/MS method to measure Myriocin in minute specimens of animal tissue. The chemical analog 14-OH-Myriocin is used as the internal standard. The two molecules are extracted from the tissue homogenate by solid-phase extraction, separated by gradient reverse-phase liquid chromatography and measured by negative ion electrospray mass spectrometry in the triple quadrupole. Detection is accomplished by Multiple Reaction Monitoring, employing the most representative transitions: 400@104 and 402@104 for Myriocin and 14-OH-Myriocin, respectively. The typical LoD and LLoQ of the optimized method are 0.9 pmoles/mL (approx. 0.016 pmoles injected) and 2.3 pmoles $/ \mathrm{mL}$, respectively, and the method is linear up to 250 pmoles $/ \mathrm{mL}$ range $\left(r^{2}=0.9996\right)$. The intra-and between-day repeatability affords a $\mathrm{CV} \% \leq 7.0$. Applications included quantification of Myriocin in mouse lungs after $24 \mathrm{hrs}$ from administration of $\sim 4$ nmoles by intra-trachea delivery. Measured levels ranged from 4.11 (median; 2.3-7.4 IQR, $\mathrm{n}=4$ ) to 11.7 (median; 7.6-22.7 IQR, $\mathrm{n}=6$ ) pmoles/lung depending on the different formulations used. Myriocin was also measured in retinas of mice treated by intravitreal injection and ranged from $0.045(<\mathrm{LoD})$ to 0.35 pmoles/retina.
\end{abstract}

Supplementary Key-words: Ceramides; Drug monitoring; Drug screening; Inflammation; Mass spectrometry; Natural products; Solid Phase Extraction 


\section{INTRODUCTION}

Sphingolipid metabolites have structural and signalling roles, controlling cell growth, cell death, inflammation and stress responses, angiogenesis, autophagy. Sphingolipid metabolism is altered in several human diseases and its manipulation is an appealing therapeutic strategy (Hannun \& Obeid, 2011). Myriocin [(2S,3R,4R,6E)-2-amino-3,4-dihydroxy-2(hydroxymethyl)-14-oxo-6-eicosenoic acid] (Myr, or thermozymocidin or ISP-1; Figure 1) is a secondary metabolite initially isolated from some thermophilic ascomycetes (Kluepfel et al., 1972; Sailer et al., 1989) and recently found as a mycotoxin contaminant of grains (Shimshoni et al., 2013). Myriocin is one of the few substances known to inhibit de novo synthesis of sphingosine by inactivating serine palmitoyl transferase (SPT) with a complex mechanism that occurs in two steps, each of which has recently been elucidated. In the first step it behaves as a classical intermediate mimic inhibitor of the substrate at the active site. Then, the co-complex degrades via an unexpected 'retro-aldol-like' cleavage mechanism to a long chain aldehyde which, in turn, irreversibly reacts with the key catalytic residue Lys265, acting as a "suicide inhibitor" of SPT (Wadsworth et al., 2013).

Myriocin has been extensively studied in different in vivo and in vitro experimental models as a tool to modulate sphyngolipids production. In particular, it seems to be is a promising candidate to treat diseases such as diabetes (Ussher et al., 2011; Kurek et al., 2017) retinitis pigmentosa (Piano et al., 2013; Strettoi et al., 2010), cystic fibrosis (Caretti et al., 2014; Caretti et al., 2016) and myocardial infarction (Reforgiato et al., 2016). Myriocin is active at few nanomoles dose (1-5 microg) as a powerful immunosuppressant, and may have clinical potential in organ transplantation and for the treatment of autoimmune diseases (Fujita et al., 1994).

Structurally, Myriocin features a carbonyl-modified polymethylene hydrophobic tail and a complex, multifunctional head group that carries two hydroxyl groups and the amino acid substructure of serine (Aragozzini et al., 1972). This complex of conflicting chemical properties (a zwitterionic, tri-hydroxylated hydrophilic "head" and a hydrophobic "tail") imparts to the molecule a limited solubility in water and in common organic solvents, a factor that complicates chemical manipulations and administration of the drug (Caretti et al., 2014, Gasco \& Gasco, 2007).

The development of pharmaceutical agents needs the availability of quantitative analytical methods, adequate for sensitivity to the expected levels in the relevant biological systems and 
for selectivity to the foreseen complexity of biological matrices. The few analytical methods so far published for Myriocin use HPLC-light scattering detection (Wang et al., 2009) or HPLC-fluorescence detection with the need of a complex pre-column derivatization with fluorenyl-methyl-chloroformate (FMOC) (Yu et al., 2009). These approaches do not attain sufficiently high specificity and low detection limits in biological samples that are necessary for further progress in the regulated pharmacological and pharmaceutical development of this promising candidate drug.

Myriocin was briefly cited in a recent article on the occurrence of mycotoxins in corn and wheat silage in Israel, detected with a liquid chromatography-tandem mass spectrometry (LCMS/MS) multi-mycotoxin method. The article only reports a value for the LoD of Myriocin $(15 \mu \mathrm{g} / \mathrm{Kg})$ and briefly comments on its potential significance as a contaminant of ruminant feed. The authors do not report analytically useful information that may facilitate measurement of Myriocin also in other matrices (Shimshoni et al., 2013). Furthermore, Myriocin was never mentioned in earlier articles that describe multi-residue analyses of several tens of fungal metabolites that possibly contaminate grains and wheat (Sulyok et al., 2006; Sulyok et al., 2007a, 2007b).

In view of the potentiality of this molecule in clinical research, we have developed and validated an assay for Myriocin quantification by LC-MS/MS. The method was at first applied to the titration of a novel solid-lipid nanoparticles-based Myriocin preparation (SLNMyriocin), and then to the quantification in different biological samples.

\section{EXPERIMENTAL}

\subsection{Reagents and Chemicals}

Methanol, ethanol, acetonitrile and formic acid (all LC-MS reagent grade) were supplied from Merck (Darmstadt, Germany). Water was MilliQ-grade. Standard Myriocin was purchased from Fermentek LTD (Jerusalem, Israel), and used as received. 14-HydroxyMyriocin (14-OH-Myr) was prepared in our laboratory by chemical reduction of the carbonyl group, purified, fully characterized by mass spectrometry and used as such. The relevant experimental details are reported in the Supplementary Materials (see online version). Myriocin loaded-solid lipid nanoparticles (SLN-Myriocin) used for mice treatment was supplied by Nanovector srl, Torino, Italy, at a nominal concentration $1 \mathrm{mM}$, and prepared for 
administration as already described (Gasco \& Gasco, 2007). Myriocin extraction and purification from biological matrix was performed on StrataTM-X $33 \mu \mathrm{m}$ Polymeric Reversed Phase SPE $30 \mathrm{mg} / 1 \mathrm{~mL}$ extraction cartridges (Phenomenex, Anzola Emilia, Italy) connected to Visiprep Solid Phase Extraction Vacuum Manifolds (Supelco, Bellefonte, USA).

\subsubsection{Myriocin and 14-OH Myriocin characterization by mass spectrometry}

Structural confirmation of standard Myriocin and of 14-OH-Myriocin was accomplished in both mass spectrometry polarities, by recording the triple quadrupole fragment mass spectra of the protonated $\left(\mathrm{MH}^{+}\right)$and deprotonated $\left([\mathrm{M}-\mathrm{H}]^{-}\right)$species over a range of nominal collision energy from 0 to $50 \Delta \mathrm{V}$. The relevant experimental details are reported in the Supplementary Materials (see online version).

\subsection{Myriocin standard solutions and calibrators}

Myriocin powder $(4.5 \mathrm{mg})$ was weighted and dissolved in ethanol $(50 \mathrm{~mL})$ by warming up at $40^{\circ} \mathrm{C}$, to a final concentration of $225 \mu \mathrm{M}$. This stock solution was diluted to an intermediate $25 \mu$ moles/L concentration in acetonitrile:formic acid (50:50, v:v). These solutions were kept at $-80{ }^{\circ} \mathrm{C}$ for 12 months and were found to be stable (data not shown). Each month, the intermediate solution was diluted to $2.5 \mu$ moles/L in acetonitrile:formic acid (50:50, v:v) and, at occurrence, the final working solution 1 (WS1) was prepared by 1:10 dilution in acetonitrile: $\mathrm{H}_{2} \mathrm{O}(50: 50$, v:v). By 1:1 serial dilutions of WS1, all the working solutions were prepared as reported in Table 1.

\subsubsection{Calibration standards in mobile phase}

For the construction of the standard curve, a $100 \mu \mathrm{L}$ aliquot of each working solution (WS) was transferred into the HPLC vials, $50 \mu \mathrm{L}$ of 250 pmoles/mL solution of $14-\mathrm{OH}-\mathrm{Myriocin}$ (12.5 pmoles) (acetonitrile: $\mathrm{H}_{2} \mathrm{O}$ 1:1) was added as Internal Standard and $10 \mu \mathrm{L}$ of the resulting solution were injected into the LC-MS/MS. The on-column final amounts are reported in Table 1.

\subsubsection{Calibration standards in biological matrices}

Series of calibrators were prepared for each analysed matrix (cultured cells, lung tissue, retina, SLNs), at the concentration judged relevant from pilot experiments. E. g., calibration samples of Myriocin in mouse lung homogenate were prepared by spiking $100 \mu \mathrm{L}$ (approx. $0.5 \mathrm{mg}$ proteins) of a control lung homogenate (approx. $10 \mathrm{mg}$ prot $/ 2 \mathrm{~mL}$ PBS) with $100 \mu \mathrm{L}$ 
of each WS calibrator and $50 \mu \mathrm{L}$ of the internal standard solution (12.5 pmoles). These samples underwent the same purification protocol of the samples as described below.

\subsection{Myriocin extraction and purification from biological matrix by SPE}

An aliquot of tissue homogenate (100 $\mu \mathrm{L}$ approx. $0.5 \mathrm{mg}$ prot or less) was added with $50 \mu \mathrm{L}$ of internal standard (12.5 pmoles), diluted to $0.5 \mathrm{~mL}$ with water and subjected to 5 sonication cycles (5 sec at amplitude 3/20 + $5 \mathrm{sec}$ in ice) (Misonix XL2000 Microson Ultrasonic Cell Disruptor XL 2000). After dilution to $1 \mathrm{~mL}$, it was loaded onto a pre-activated SPE columns. The disposable tubes were connected to the Visiprep Solid Phase Extraction Vacuum Manifolds and, after vacuum application with a water pump, pre-activated by washing with 1 $\mathrm{mL}$ of methanol followed by $1 \mathrm{~mL}$ of water. The vacuum was disconnected from the Visiprep apparatus, the diluted samples were loaded, and the vacuum was applied again, not exceeding the flow rate of $1 \mathrm{~mL} / \mathrm{min}$. Two washings were performed with water:methanol 85:15 (by volume, $1 \mathrm{~mL}$ ) and water:methanol 50:50 (by volume, $1 \mathrm{~mL}$ ), then the columns were completely dried by flushing air for $4 \mathrm{~min}$. The vacuum was disconnected and the $12-\mathrm{mL}$ polypropylene tubes used to collect washings were discharged and replaced with $5 \mathrm{~mL}$ glass disposable tubes to collect the sample fraction. Myriocin and internal standard were recovered under vacuum by applying $0.5 \mathrm{~mL}$ of methanol to the dried SPE cartridges. After evaporation under nitrogen, samples were stored at $-20{ }^{\circ} \mathrm{C}$ or directly analysed. Before LCMS analysis, $150 \mu \mathrm{L}$ of $50 \%$ water:acetonitrile were added, the extract transferred to the HPLC vial and $10 \mu \mathrm{L}$ injected for analysis.

\subsection{Spike recovery and matrix effect}

The recovery of spiked Myriocin in the examined biological matrices (cultured cells, lung, retina) was determined by comparing the analytical results of this measurement to the unextracted standard curve used for the linearity study. Three replicates were prepared for each concentration level. The biological matrices that were examined in this preliminary phase of pharmacological study (cultured cells, lung, retina) are very "soft" tissues and it is deemed that complete incorporation of the Myriocin spike and equilibration of the internal standard occurs within the typical sample equilibration time of approx. 10-18 h.

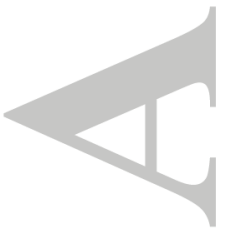




\subsection{Analytical conditions}

\subsubsection{Instrumentation}

The analytical system consisted of a HPLC coupled to a tandem mass spectrometer. The liquid chromatograph system is a Dionex 3000 UltiMate instrument with autosampler, binary pump, and column oven (Thermo Fisher Scientific, USA). The tandem mass spectrometer is an AB Sciex 3200 QTRAP instrument with electrospray ionization (ESI) TurboIonSpray ${ }^{\mathrm{TM}}$ source (AB Sciex S.r.l., Milano, Italy). Instruments were managed with proprietary manufacturers' software and according to manufacturers' instructions. The analytical data were processed by Analyst software (version 1.4).

\subsubsection{Optimized analytical chromatographic conditions}

Myriocin and 14-OH-Myriocin were separated on a reversed-phase column Inertsil ODS3, $150 \times 3.0 \mathrm{~mm}$ i.d., $3 \mu \mathrm{m}$ particle size (GL Sciences, Tokio, Japan), with a linear gradient between eluent $\mathrm{A}$ (water $0.1 \%$ formic acid) and eluent $\mathrm{B}$ (acetonitrile). The column was equilibrated with 50:50 A:B, then eluent $\mathrm{A}$ was increased to $100 \%$ in 5 min and brought back to $50 \%$ in $1 \mathrm{~min}$. After additionally $2 \mathrm{~min}$ at 50:50, the analysis was stopped. The mobile phase was delivered at $0.4 \mathrm{~mL} / \mathrm{min}$, the autosampler and the column oven were kept at $20^{\circ} \mathrm{C}$ and $45^{\circ} \mathrm{C}$, respectively.

\subsubsection{Optimized analytical mass spectrometry conditions}

Detection was accomplished in the negative ion mode by multiple reaction monitoring (MRM) in the triple quadrupole tandem mass spectrometer. Only one analytically useful transition could be identified and was used: m/z $400\left([\mathrm{M}-\mathrm{H}]^{-}\right)$to m/z 104 for Myriocin, and $\mathrm{m} / \mathrm{z} 402\left([\mathrm{M}-\mathrm{H}]^{-}\right)$to $\mathrm{m} / \mathrm{z} 104$ for the internal standard 14-OH-Myriocin, respectively (Figures S3B and S5B for the tandem mass spectra; Figure S6 for the breakdown curves). Dwell time was set as $350 \mathrm{msec}$, for both transitions. The final optimized conditions for quantitation by MS/MS detection in negative ion mode for Myriocin and IS are reported in Table 2. Quadrupole Q1 and Q3 were set on unit resolution.

\subsection{Validation}

\subsubsection{Linearity}

The linearity of calibration curve was calculated by unweighted least squared linear regression analysis of the (area Myr/area IS ratio vs Myr concentration data set). The Pearson $r^{2}$ coefficient and $p$-value were used as assessors of linearity. 


\subsubsection{Sensitivity and detection limits}

The Limit-of-Detection (LoD, defined as three times the value of the intercept) was calculated from the regression parameters (see above). The Lower-Limit-of-Quantification (LLoQ) was estimated as the concentration of the calibrator that yielded back-calculated concentration at $\pm 20 \%$ of the nominal value (accuracy) and a Coefficient-of-Variation lower than $20 \%$ (precision on three replicates).

\subsubsection{Measurement specificity}

The specificity of the method was verified by analysing extracts from six lung samples from control mice and checking for the absence of spurious signal at the LC peak region for Myriocin and IS. The responses of the interfering peak or background noise at the retention time and transition of the internal standard (14-OH-Myriocin) was deemed as acceptable if its intensity was less than $5 \%$ of that of the added internal standard (12.5 pmoles, 0.83 pmoles injected).

The possible presence of interference at the retention time and detection channel of Myriocin was assessed by recalculating the calibration line with the standard addition method (see Supplementary). The possibility to appreciate small amount of Myriocin in specific matrix was judged acceptable if the value of the recalculated interference was lower than $50 \%$ of the LoD.

\subsubsection{Precision and Accuracy of determinations in specimens}

Samples for the determination of precision and accuracy were prepared by spiking control matrix batches with Myriocin at appropriate concentrations within the calibration range. All the samples were stored at $-20^{\circ} \mathrm{C}$. The intra-assay precision and accuracy were estimated on three replicates (acceptance criteria as above) analysed in the same day, the inter-assay precision was calculated on three replicates analysed within a 10-weeks period.

\subsubsection{Stability of extracts during analysis (in-Run Stability)}

The stability of Myriocin and IS in the injection solvent was determined for 5 hours in the auto-sampler kept at $10{ }^{\circ} \mathrm{C}$ and at $20{ }^{\circ} \mathrm{C}$. The peak-areas of the analyte and IS obtained at the start of the analytical batch were used as the reference to determine the stability at subsequent points. 


\subsection{Myriocin-loaded SLNs titration}

Myriocin-SLN stock solution supplied $1 \mathrm{mmol} / \mathrm{L}$ as reported (Strettoi et al., 2010), was checked by LC-MS/MS for Myriocin content. Three aliquots ( $5 \mu \mathrm{L}=5000$ pmoles $)$ from six independent vials of the $1 \mathrm{mmol} / \mathrm{L}$ preparation were withdrawn under vigorous stirring and diluted 1:200 (v:v) with acetonitrile. After vortexing and centrifugation for $5 \mathrm{~min}$ at 12000 rpm, $100 \mu \mathrm{L}$ were diluted 1:20 with acetonitrile: $\mathrm{H}_{2} \mathrm{O}$ 1:1 to a nominal concentration of 250 pmoles/mL. A $100 \mu \mathrm{L}$ aliquot (25 pmoles) was transferred into the HPLC vial, added with 50 $\mu \mathrm{L}$ internal standard (12.5 pmoles) and $10 \mu \mathrm{L}$ injected into the HPLC.

\subsection{Mice treatment and lung preparation}

Animals were handled according to Italian laws, internal University regulations and, for the retina study (vide infra) following the Association for Research in Vision and Ophthalmology (ARVO) statement for the use of animals in research.

To validate the method for Myriocin quantification in tissues, a pilot experiment was carried out following, with some modifications, the protocol used by Caretti et al. (Caretti et al., 2014), who tested the anti-inflammatory action of Myriocin as a therapeutic potential in cystic fibrosis. Myriocin was prepared in 10\% DMSO-sterile saline solution at $42.0 \mu \mathrm{M}$, alternatively SLN-Myriocin was prepared $62.5 \mu \mathrm{M}$ by $1: 16$ dilution in sterile saline of the 1 $\mathrm{mM}$ stock.

Briefly, C57BLK6 mice (male and female 9 weeks age, $\approx 30$ gr each), were housed in filtered cages and permitted unlimited access to food and water. . A deep stage of intra peritoneal anaesthesia was accomplished with Avertin (2,2,2,-tribromoethanol in 1\% tert-amyl alcohol; both reagents from Sigma-Aldrich, Milan). A twentyfive/thirty g C57BLK6 mouse was treated i.p. with $500 \mu \mathrm{L}$ of a solution of Avertin $(10 \mathrm{mg} / \mathrm{mL}$ in saline $)$ when the animal had to be raised from anaesthesia; for sacrifice the animal was treated with $800 \mu \mathrm{L}$ of the same solution. One anaesthesia was reached, the animals were treated with $75 \mu$ of Myriocin either dissolved in DMSO (1.26 $\mu \mathrm{g}, 3.15$ nmoles) or uploaded in SLN (1.87 $\mu \mathrm{g}, 4.69$ nmoles), by intra-trachea instilled by means of MicroSprayer® Aerosolizer -Model IA1C, attached to "FMJ-250 High Pressure Syringe" (Penn-Century Inc., US) (Caretti et al., 2014; Caretti et al., 2016). Control animals were treated with the corresponding empty vehicle. Twenty-four $h$ after treatment, animals were euthanized; lungs were perfused with PBS and homogenized in 
$2 \mathrm{ml}$ of PBS containing protease inhibitors (Roche Italia, Italy) with Ultraturrax. After measuring homogenate total protein content, specific volumes corresponding to equal amount of proteins $(\approx 0.5 \mathrm{mg})$, were directed to Myriocin and ceramides purification protocols before LC-MS/MS analysis.

\subsection{Mice treatment and retina preparation}

To run a pilot analytical experiment, wild-type mice handled according to Italian laws and following the Association for Research in Vision and Ophthalmology (ARVO) statement for the use of animals in research. Mice ( $n=3$; 3-months-old; Jackson Laboratories strain $\mathrm{C} 57 \mathrm{Bl} / 6 \mathrm{~J}$,) were kept in a local facility with water and food ad libitum in a 12-h light/dark cycle with illumination level below 60 lux. The treatment protocols approved by the Italian Ministry of Veterinary Health (Protocol \#14/D-2014, CNR Neuroscience Institute) involved intravitreal injection of $1 \mu \mathrm{l}$ of a $1.88 \mathrm{mM}$ solution of Myriocin in DMSO (0.94 nmoles), carried out under Avertine general anaesthesia, as described in (Strettoi et al., 2010). An identical volume of DMSO vehicle was injected into the opposite eye of the same animal to obtain control samples for analytical development. Mice were sacrificed after 4 hours from the single injection, the retinas were separated from the pigmented epithelium and the choroid and collected in ice-cold, oxygenated ACSF (Artificial cerebrospinal fluid), frozen at $\mathrm{C}^{\circ}-80$ and subsequently delivered to the analytical laboratory. Specimens was thawed and manually homogenized in PBS containing protease inhibitors. This homogenate followed the same procedure described above for lung samples.

\subsection{Calculations and statistical elaboration}

Peak areas were obtained from the proprietary instrument's application software MultiQuant, and transferred to Excel spreadsheets to be further elaborated. A custom Excel spreadsheet was used to calculate line parameters (intercept, slope and associated standard errors), backcalculate analytical concentration and associated errors, essentially according to and fully validated against a calculated example of Miller\&Miller's textbook (1984). Initial statistical analysis of results was performed using the GraphPad Prism version 7.00 for Windows, GraphPad Software, La Jolla California USA, www.graphpad.com. Data are expressed as mean \pm SEM or as median and interquartile range, according to sample numbers and end-user necessities. To assess the significance of the differences between treatments, the nonparametric Mann-Whitney test was used. Statistical significance was assumed at $\mathrm{p} \leq 0.05$. 


\section{RESULTS AND DISCUSSION}

\subsection{Preparation and characterization of compounds}

Since only Myriocin is a commercially available chemical standard, we chose to set up the analytical method for its quantification using 14-OH-Myriocin, the simplest candidate internal standard that could be easily prepared and characterized by mass spectrometry in our laboratory (see Supplementary). As apparent from the integrated fragment mass spectra in Figure S3B and S5B of Supplementary Materials, both for Myriocin and for 14-OHMyriocin, the multi-functional polar head-group tightly holds the charge in both polarities and drives the fragmentation. The functional groups in the middle of the hydrocarbon chain essentially do not modify the spectrum and, in any case, do not yield useful fragments for analyte quantification. A thorough assignment of the fragmentation pattern for both compounds is reported in the Supplementary Materials.

\subsection{Mass Spectrometry operating conditions}

Based on the spectroscopic behaviour of Myriocin and 14-OH-Myriocin, detection was accomplished by multiple reaction monitoring (MRM) in the triple quadrupole tandem mass spectrometer in the negative ion mode. Only one analytically useful transition could be identified and was used: m/z 400 ([M-H] $\left.]^{-}\right)$to m/z 104 for Myriocin, and m/z $402\left([\mathrm{M}-\mathrm{H}]^{-}\right)$to $\mathrm{m} / \mathrm{z} 104$ for the internal standard 14-OH-Myriocin, respectively (Figures S3B and S5B for the tandem mass spectra; Figure S6 for the breakdown curves). Dwell time was set as $350 \mathrm{msec}$, for both transitions. The final optimized conditions for quantitation by MS/MS detection in negative ion mode for Myriocin and IS are reported in Table 2. Quadrupole Q1 and Q3 were set at unit resolution.

\subsection{Chromatography operating conditions}

Among different ODS column tested, only the Inertsil ODS-3 column modified with octadecyl groups and endcapped provided strong hydrophobic interactions and retained both Myriocin and 14-OH-Myriocin with a baseline separation (Figure 2) and a good peak shape. Neither Myriocin, nor 14-OH-Myriocin displayed high solvent noise level under the final conditions reported, while replacing acetonitrile with methanol or adding buffers or modifiers like ammonium acetate or tetrahydrofurane severely worsened the chromatographic quality (data not shown). In our system, the gradient did not produce interfering humps and peaks and allowed a sample-to-sample analysis time of 9 min, including gradient reconditioning. 
Attempts to decrease the reconditioning time worsened analysis reproducibility. We did not test a more performing column geometry ( $2 \mu \mathrm{m} \times 2 \mathrm{~mm} \times 50 \mathrm{~mm}$, for instance), but in view of Myriocin introduction in clinical protocols and pharmacokinetics studies, this transfer will be explored, whenever necessary.

\subsection{Calibration curves}

Figure 3 shows examples of calibration curves constructed from pure standards in the widest (0-250 pmoles $/ \mathrm{mL})$ calibration range useful for the analysis of samples supplied to the laboratory (left panel) and from lung homogenate in the calibration range of actual samples (right panel) are reported. The standard curve constructed on pure standard showed linearity up to 250 pmoles $/ \mathrm{mL}$, while above this level solubility issue could be the major factor for unmanaged variability of individual chromatographic runs. Typical regression coefficients were consistently better than 0.9998 . No significant difference $(\mathrm{p}<0.05)$ was found between slopes of extracted and non-extracted curves over the extended calibration range. To improve the quality of analytical results in the biological samples submitted to the laboratory, the curves constructed in the individual tested matrices were restricted to the concentration range of actual samples (0-30 pmoles/mL; right panel; see also Figure S8 in Supplementary). Under this condition, the LoD and LLoQ were 0.9 pmoles $/ \mathrm{mL}$ and 2.3 pmoles $/ \mathrm{mL}$, respectively.

\subsection{Extraction Recovery and Matrix effect}

Each analyzed biological matrix showed the presence of interfering signal at retention time and detection transition of Myriocin also in blank specimens that were used to construct the calibration curve and to set-up the pre-analytical preparation steps. The recovery of Myriocin was quantitative at all concentrations in the $2.0-250$ pmoles $/ \mathrm{mL}$ range $(106 \pm 15 \%$ for the extracts of cultured cells, similar results for the other biological tissues). In the lower range, recovery was deceivably higher due to the presence of an interfering signal that also reflected in the positive intercept of the calibration curve. Full documentation is reported in the Supplementary (Table S2).

To enhance the possibility of quantify Myriocin also in specimens at very low concentration, the standard addition method was used. A comparison of the results obtained, for two specific and critical specimens (single and pooled murine retinae at value close to the LLoQ), with the

standard curve and with the standard addition method yielded an agreement lower than $30 \%$ (2.4 vs. 3.7 pmoles $/ \mathrm{mL}$, respectively), thus suggesting that the standard curve yielded a higher intercept than the individual specimens did. Due to the lengthier procedure, standard addition 
is reserved to the re-analysis of samples for which the assignment of an upper-limit value is critical to the pharmacologic research.

\subsection{Intra-day and Inter-day Precision and Accuracy}

Table 3 summarizes intra- and inter-day accuracy and precision of Myriocin measurement in lung homogenate, since this is the most abundant tissue available in the laboratory. To keep the temperature of the sample rack of the automatic injector close to room temperature $\left(20^{\circ}\right.$ C) is of a fundamental importance to achieve repeatability of the analysis (See Supplementary Materials).

\subsection{Applications}

Among the several different pilot application of this method that are currently developed in the laboratory, selected examples on different matrices are reported, to demonstrate the general applicability of the core method and the flexibility in accepting matrix specific modification.

\subsubsection{SLN-Myriocin titration}

The present method was applied to the exact titration of the commercial SLN-Myriocin specifically prepared to study Myriocin biochemical activity in animal models. This preparation was supplied at a nominal concentration $1 \mathrm{mM}$ (Gasco \& Gasco, 2007), and previously characterized by TLC (Strettoi et al., 2010). Three aliquots from six independent vials were processed as described in Material and Methods, and injected in triplicate. The results are reported in Table 4 and show a Myriocin concentration in SLN preparation ranging from 0.6 to 1.13 with a mean of $0.78 \mathrm{mM}$. The poor homogeneity of the solid lipid preparation probably account for the observed variability.

\subsubsection{Myriocin quantification in mice lung tissue}

This first pilot study on mice was aimed to validate the use of the developed LC-MS/MS method for "in vitro" and "in vivo" studies on Myriocin concentration/activity relationship. The drug was successfully quantified in mice lungs after $24 \mathrm{~h}$ from intra-tracheal administration of two different formulations. In particular, the left panel of Figure 4 shows that residual Myriocin is higher after delivery in DMSO (11.7; 7.6-22.7 median; IQR, $\mathrm{n}=6$ pmoles/lung) than after delivery in $\operatorname{SLN}$ (4.11; 2.3-7.4 median; IQR, $\mathrm{n}=4$ pmoles/lung). These residual concentrations corresponding to $0.89 \pm 0.12$ pmoles $/ \mathrm{mg}$ prot; mean $\pm \mathrm{SEM}$, and to $0.26 \pm 0.05 \mathrm{pmoles} / \mathrm{mg}$ prot; mean \pm SEM, respectively, i.e. $0.23 \%$ and $0.10 \%$ of the initial 
dose. A few samples from mice treated with SLN-Myriocin yielded Myriocin concentration between the LoD and the LLoQ of the method. More accurate measurement of Myriocin concentration in these specimens may be in principle achieved by analysing larger aliquots (200 $\mu \mathrm{L}$ of homogenate) whenever necessary. No appreciable signal was detected in the Myriocin $\mathrm{m} / \mathrm{z}$ trace after treatment with either DMSO or empty SLN.

To exemplify the use of the combined measurement of Myriocin as the pharmacological agent and of ceramides as the influenced biochemical indicator, right panel of Figure 4 shows the main ceramide species measured separately by mass spectrometry (Munoz-Olaya et al., 2008) in the same lung homogenates. Both DMSO-Myriocin, and SLN-Myriocin produced a significant decrease in ceramide concentration $v s$ the respective control animals. Moreover, when Myriocin was delivered enclosed in SLN, the decrease of ceramide concentration was higher than with DMSO formulation (decrease $29.6 \%$ and $54.8 \%$ vs controls, MyriocinDMSO and Myriocin-SLN, respectively).

This pilot experiment leads to preliminary conclusions: (i) despite a roughly equivalent dose, SLN-Myriocin is likely more efficiently delivered in lung than DMSO-Myriocin; (ii) delivery with SLN may cause a higher absorption and thus a greater therapeutic effect. This phenomenon is demonstrated by the coincidence of the two measured effects, as lowering of ceramides concentration and as higher biotransformation of the Myriocin molecule, which is the molecular mechanism of its action.

\subsubsection{Myriocin quantification in mouse retina}

To test the ability of the developed method to deal with biological specimens available only in minute amount such as for the study of Retinitis Pigmentosa (see Materials and Methods), retinas of wild type mice $(\mathrm{n}=3)$ treated by intravitreal administration with DMSO-Myriocin were excised after $4 \mathrm{hrs}$ from injection and assayed. The retinas yielded a total Myriocin amount of 0.045 (just below the LoD), 0.12 and 0.35 pmoles, respectively corresponding to $0.33,0.87$ and 2.3 pmoles/mg prot. To test the accuracy of the measurement in an individual retina, the third sample of this series (corresponding to $\approx 0.15 \mathrm{mg}$ prot and weighting less than $1 \mathrm{mg}$ ), was repeatedly analysed $(\mathrm{n}=9)$ and yielded a mean Myriocin amount of $0.35 \pm 0.02$ pmoles $(\mathrm{CV} 6.7 \%)$. It is interesting to note that the untreated contralateral retinas, individually assayed, did not yield Myriocin chromatographic signals. 


\section{CONCLUSIONS}

This article describes in detail for the first time a method for the measurement of Myriocin in biological specimens by liquid chromatography and mass spectrometry. LC-MS/MS is considered the technique of choice in organic trace bioanalysis, due to its high level of molecular specificity, high sensitivity and the possibility to deal with multiple pharmaceutical and biological specimens, such as Lipid Solid Nanoparticle formulations, cell cultures and tissue homogenates available only in minute amounts.

The established method results in a rapid, accurate, precise, sensitive procedure, and the presented report shows examples of useful measurements performed also on minute samples, such as individual murine retinae. Specimens of this type derive from ongoing experimental and pre-clinical studies on diseases in which the production and degradation of ceramides is possibly involved.

There is a potential for improvement of this method, which will be pursued, as the importance of Myriocin as a candidate drug for the treatment of otherwise untacked diseases will emerge. One such improvement is the use of a suitable stable-isotope labelled form of Myriocin as the internal standard for quantification. Spectroscopic studies, of which only preliminary results are reported in the Supplementary materials, highlight that the $13,13^{\prime}, 15,15^{\prime}$ tetra-deuterated analogue is the most accessible stable isotope isoform, and a viable candidate isotope labelled internal standard. As a further advancement, the use of highresolution mass spectrometry will likely overcome the emerged problem of a low-level interference and further lower the detection limit.

Last, the results from the pilot study show that, as expected, the intra-tracheal treatment with Myriocin decreases ceramides concentration in the lungs. It is to note that when Myriocin is administered in the lungs as SLN instead as the "soluble" form in DMSO, a lower amount (approximately one half) is actually recovered from the organ after $24 \mathrm{hrs}$. This may suggest that the nanocarriers allow a higher intracellular uptake, systemic distribution and/or a faster excretion. Up to now very little is known about Myriocin metabolic behavior "in vivo" due to the lack of suitable analytical method for its quantitation in biological matrices. To the best of our knowledge, this is the first report of a method that enable the detection of minimal efficacious dose of the compound even starting from minute samples. To elucidate its metabolic fate, in addition to the LC-MS/MS method to quantify the Myriocin parent 
molecule, could be interesting to develop other procedures to identify degradation products occurring during its metabolic pathway (e.g. 2-hydroxyl-C 18 aldehyde species).

Several investigations will be feasible thanks to the availability of this analytical method such as the study of Myriocin bioavailability and catabolism, the dose/effect relationship and the performance of different formulations and administration routes. This knowledge will be useful in view of its prospective use for therapeutic purpose in human pathologies.

FUNDING: This work was supported by a Grant from Fondazione Roma, Roma (Italy), Prot. 106/A1 "Slowing down Retinits Pigmentosa with a mutation-independent approach: in vivo assessment on multiple animal models".

G.M.C. and J.R. are supported by the institutional grants of the University of Milan and by $\mathrm{PhD}$ program in "Molecular and Translational Medicine" and "Biochemical Sciences" of the University of Milan, Italy.

A.C is supported by Institutional grants from Department of Health Sciences, University of Milan, Milan, Italy.

Conflict of Interest: The authors declare that they have no conflict of interest.

Supplementary materials to: Campisi et al., "Determination of the serine palmitoyltransferase inhibitor Myriocin by electrospray and Q-trap mass spectrometry." 


\section{REFERENCES}

Aragozzini, F., Manachini, P. L., Craveri, R., Rindone, B., \& Scolastico, C. (1972). Structure of thermozymocidin. Experientia, 28(8), 881-882.

Caretti, A., Bragonzi, A., Facchini, M., De Fino, I., Riva, C., Gasco, P., Signorelli, P. (2014). Antiinflammatory action of lipid nanocarrier-delivered myriocin: therapeutic potential in cystic fibrosis. Biochim Biophys Acta, 1840(1), 586-594.

Caretti, A., Torelli, R., Perdoni, F., Falleni, M., Tosi, D., Zulueta, A., Signorelli, P. (2016). Inhibition of ceramide de novo synthesis by myriocin produces the double effect of reducing pathological inflammation and exerting antifungal activity against $\mathrm{A}$. fumigatus airways infection. Biochim Biophys Acta, 1860(6), 1089-1097.

Fujita, T., Inoue, K., Yamamoto, S., Ikumoto, T., Sasaki, S., Toyama, R., .Okumoto, T. (1994). Fungal metabolites. Part 11. A potent immunosuppressive activity found in Isaria sinclairii metabolite. J Antibiot (Tokyo), 47(2), 208-215.

Gasco, M. R., \& Gasco, P. (2007). Nanovector. Nanomedicine (Lond), 2(6), 955-960.

Hannun, Y. A., \& Obeid, L. M. (2011). Many ceramides. J Biol Chem, 286(32), 27855-27862.

Kluepfel, D., Bagli, J., Baker, H., Charest, M. P., \& Kudelski, A. (1972). Myriocin, a new antifungal antibiotic from Myriococcum albomyces. J Antibiot (Tokyo), 25(2), 109-115.

Kurek, K., Garbowskam, M., Ziembicka, D.M., , B., Rogowski, J., Chabowski, A., Górski. J., Żendzian-Piotrowska, M. Myriocin treatment affects lipid metabolism in skeletal muscles of rats with streptozotocin-induced type 1 diabetes. Adv Med Sci. 2017 Mar;62(1), 65-73. doi: 10.1016/j.advms.2016.04.003. Epub 2016 May 9.

Piano, I., Novelli, E., Gasco, P., Ghidoni, R., Strettoi, E., \& Gargini, C. (2013). Cone survival and preservation of visual acuity in an animal model of retinal degeneration. Eur J Neurosci, 37(11), 1853-1862.

Reforgiato, M. R., Milano, G., Fabrias, G., Casas, J., Gasco, P., Paroni, R., .Signorelli, P. (2016). Inhibition of ceramide de novo synthesis as a postischemic strategy to reduce myocardial reperfusion injury. Basic Res Cardiol, 111(2), 12.

Sailer, M., Sasek, V., Sejbal, J., Budesinky, M., \& Musilek, V. (1989). Flavovirin--a new antifungal antibiotic produced by the pyrenomycete Melanconis flavovirens. J Basic Microbiol, 29(6), 375-381.

Strettoi, E., Gargini, C., Novelli, E., Sala, G., Piano, I., Gasco, P., \& Ghidoni, R. (2010). Inhibition of ceramide biosynthesis preserves photoreceptor structure and function in a mouse model of retinitis pigmentosa. Proc Natl Acad Sci U S A, 107(43), 18706-18711.

Sulyok, M., Berthiller, F., Krska, R., \& Schuhmacher, R. (2006). Development and validation of a liquid chromatography/tandem mass spectrometric method for the determination of 39 mycotoxins in wheat and maize. Rapid Commun Mass Spectrom, 20(18), 2649-2659.

Sulyok, M., Krska, R., \& Schuhmacher, R. (2007a). Application of a liquid chromatography-tandem mass spectrometric method to multi-mycotoxin determination in raw cereals and evaluation of matrix effects. Food Addit Contam, 24(10), 1184-1195.

Sulyok, M., Krska, R., \& Schuhmacher, R. (2007b). A liquid chromatography/tandem mass spectrometric multi-mycotoxin method for the quantification of 87 analytes and its application to semi-quantitative screening of moldy food samples. Anal Bioanal Chem, 389(5), 1505-1523.

Ussher, J.R., Koves, T.R., Cadete, V.J., Zhang, L., Jaswal, J.S., Swyrd, S.J., Lopaschuk, D.G., Proctor, S.D., Keung, W., Muoio, D.M., Lopaschuk, G.D. Inhibition of de novo ceramide synthesis reverses diet-induced insulin resistance and enhances whole-body oxygen consumption. Diabetes. 2010 Oct;59(10):2453-64. doi: 10.2337/db09-1293. Epub 2010 Jun 3.

Wadsworth, J. M., Clarke, D. J., McMahon, S. A., Lowther, J. P., Beattie, A. E., Langridge-Smith, P. R., .. . Campopiano, D. J. (2013). The chemical basis of serine palmitoyltransferase inhibition by myriocin. J Am Chem Soc, 135(38), 14276-14285.

Wang, S., Yang, F. Q., Feng, K., Li, D. Q., Zhao, J., \& Li, S. P. (2009). Simultaneous determination of nucleosides, myriocin, and carbohydrates in Cordyceps by HPLC coupled with diode array detection and evaporative light scattering detection. J Sep Sci, 32(23-24), 4069-4076. 
Yu, J., Xu, H., Mo, Z., Zhu, H., \& Mao, X. (2009). Determination of myriocin in natural and cultured Cordyceps cicadae using 9-fluorenylmethyl chloroformate derivatization and highperformance liquid chromatography with UV-detection. Anal Sci, 25(7), 855-859.
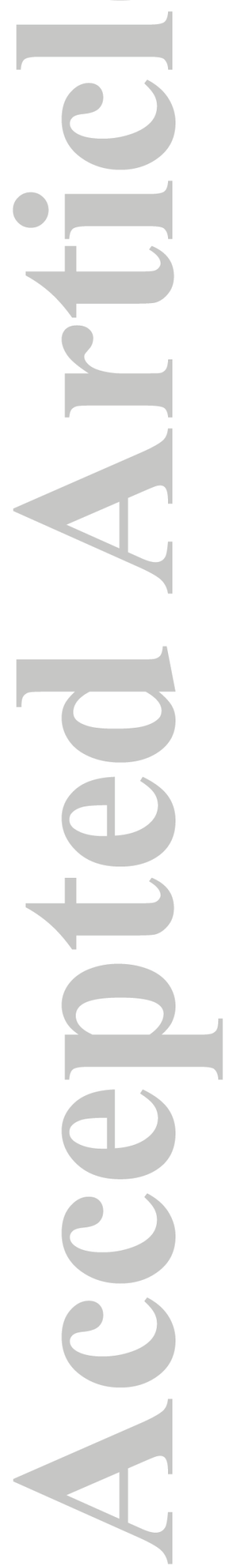
Table 1. Concentration of working solutions (WS) used for Myriocin linearity study

\begin{tabular}{|c|c|c|c|c|c|c|}
\hline & $\begin{array}{c}\text { WS concentration } \\
\text { Myriocin } \\
\text { pmoles } / \mathrm{mL}\end{array}$ & $\begin{array}{c}\text { IS } \\
\text { pmoles/mL }\end{array}$ & $\begin{array}{c}\text { in vial } \\
\text { Myriocin } \\
\text { pmoles }\end{array}$ & $\begin{array}{c}\text { IS } \\
\text { pmoles }^{\text {a }}\end{array}$ & $\begin{array}{l}\text { injected } \\
\text { Myriocin } \\
\text { pmoles }^{\text {a }}\end{array}$ & $\begin{array}{c}\text { IS } \\
\text { pmoles a }\end{array}$ \\
\hline WS1 & 250.00 & 250.00 & 25.000 & 12.5 & 1.6660 & 0.83 \\
\hline WS2 & 125.00 & 250.00 & 12.500 & 12.5 & 0.8330 & 0.83 \\
\hline WS3 & 62.50 & 250.00 & 6.250 & 12.5 & 0.4160 & 0.83 \\
\hline WS4 & 31.25 & 250.00 & 3.125 & 12.5 & 0.2080 & 0.83 \\
\hline WS5 & 15.60 & 250.00 & 1.560 & 12.5 & 0.1040 & 0.83 \\
\hline WS6 & 7.81 & 250.00 & 0.781 & 12.5 & 0.0520 & 0.83 \\
\hline WS7 & 3.91 & 250.00 & 0.391 & 12.5 & 0.0260 & 0.83 \\
\hline WS8 & 1.95 & 250.00 & 0.195 & 12.5 & 0.0130 & 0.83 \\
\hline WS9 & 0.97 & 250.00 & 0.097 & 12.5 & 0.0065 & 0.83 \\
\hline WS10 & 0.49 & 250.00 & 0.049 & 12.5 & 0.0032 & 0.83 \\
\hline WS11 & 0 & 250.00 & 0 & 12.5 & 0 & 0.83 \\
\hline
\end{tabular}


Table 2. Mass Spectrometry conditions for analysis of Myriocin and 14-OH-Myriocin

\begin{tabular}{|ll}
\hline COMMOAin gas & COM PARAMETERS \\
\hline Nebulizing gas (Gas1) & $30 \mathrm{psi}$ \\
Heater gas (Gas2) & $55 \mathrm{psi}$ \\
Collision gas (CAD) setting medium & $65 \mathrm{psi}$ \\
ESI potential & $65.2 * 10^{-5} \mathrm{torr}$ \\
Needle temperature & $-3500 \mathrm{eV}$ \\
\hline Declustering potential (DP) & $650^{\circ} \mathrm{C}$ \\
\hline Collision energy (CE) & $-40 \mathrm{~V}$ \\
Collision exit potential (CXP) & $-30 \mathrm{~V}$ \\
Collision entrance potential (CEP) & $-3 \mathrm{~V}$ \\
\hline Entrance potential (EP) & $-30 \mathrm{~V}$ \\
\hline
\end{tabular}


Table 3. Intra-and Inter-day variability

Myriocin concentration (pmoles/ml)

\begin{tabular}{|lllll}
\multicolumn{2}{l}{ Myriocin concentration $(\mathrm{pmoles} / \mathrm{ml})$} & & \\
& TIME & LOW & MEDIUM & HIGH \\
& & $\mathbf{1 5 . 6}$ & $\mathbf{6 2 . 5}$ & $\mathbf{2 5 0}$ \\
\hline INTRA-DAY & DAY1 & 0.115 & 0.410 & 1.655 \\
& & 0.125 & 0.426 & 1.613 \\
& & 0.113 & 0.409 & 1.630 \\
& Mean \pm SD & $\mathbf{0 . 1 1 8} \pm \mathbf{0 . 0 0 6}$ & $\mathbf{0 . 4 1 5} \pm \mathbf{0 . 0 0 9}$ & $\mathbf{1 . 6 3 3} \pm \mathbf{0 . 0 2 1}$ \\
& CV\% & $\mathbf{5 . 3 7 7}$ & $\mathbf{2 . 2 3 0}$ & $\mathbf{1 . 2 8 9}$ \\
\hline INTER-DAY & Week 1 & 0.104 & 0.401 & 1.619 \\
& Week 4 & 0.092 & 0.411 & 1.572 \\
& Week 10 & 0.092 & 0.386 & 1.734 \\
& Mean \pm SD & $\mathbf{0 . 0 9 6} \pm \mathbf{0 . 0 0 7}$ & $\mathbf{0 . 3 9 9} \pm \mathbf{0 . 0 1 2}$ & $\mathbf{1 . 6 4 1} \pm \mathbf{0 . 0 8 3}$ \\
& CV\% & $\mathbf{7 . 0 9 3}$ & $\mathbf{3 . 0 5 9}$ & $\mathbf{5 . 0 8 4}$
\end{tabular}

This article is protected by copyright. All rights reserved. 
Table 4. SLN-Myriocin titration by Mass Spectrometry

\begin{tabular}{lllll}
\hline & \multicolumn{4}{l}{ Aliquots measured concentration $(\mathbf{m M})$} \\
& $\mathbf{1}$ & $\mathbf{2}$ & $\mathbf{3}$ & Mean \\
\hline Vial1 & 0.94 & 0.96 & 1.00 & 0.97 \\
Vial2 & 0.61 & 0.60 & 0.62 & 0.61 \\
Vial3 & 0.59 & 0.61 & 0.60 & 0.60 \\
Vial4 & 0.74 & 0.74 & 0.76 & 0.75 \\
Vial5 & 0.59 & 0.62 & 0.69 & 0.63 \\
Vial6 & 0.86 & 1.28 & 0.98 & 1.13 \\
\hline & & & mean & $\mathbf{0 . 7 8 0}$ \\
\hline
\end{tabular}

Three aliquots from six vials were prepared as described in Materials and Methods and injected in triplicate into the Spectrometer. Vials concentration was calculated from the mean of the three aliquots. Quantification was carried out vs calibration curve in pure Myriocin standard. The expected Myriocin concentration was $1 \mathrm{mM}$. The found Myriocin concentration $(0.78 \mathrm{mM})$ was calculated as the mean value from six vials.
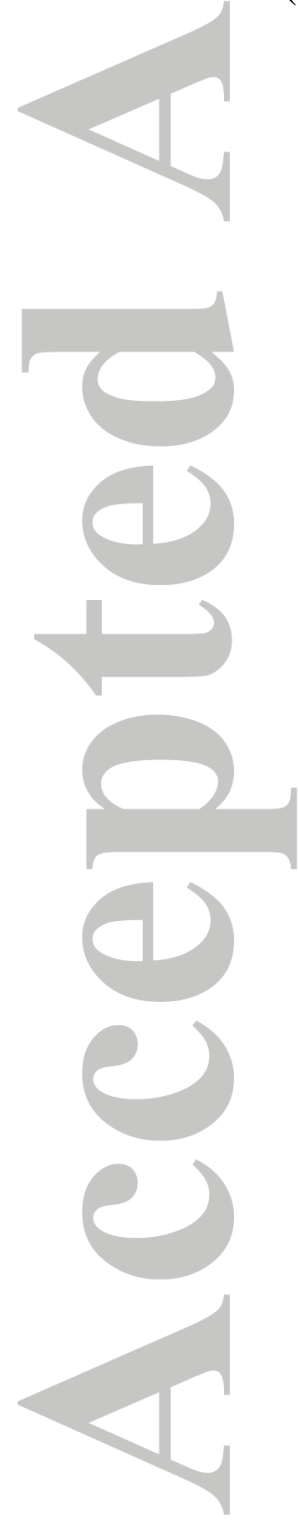

This article is protected by copyright. All rights reserved. 


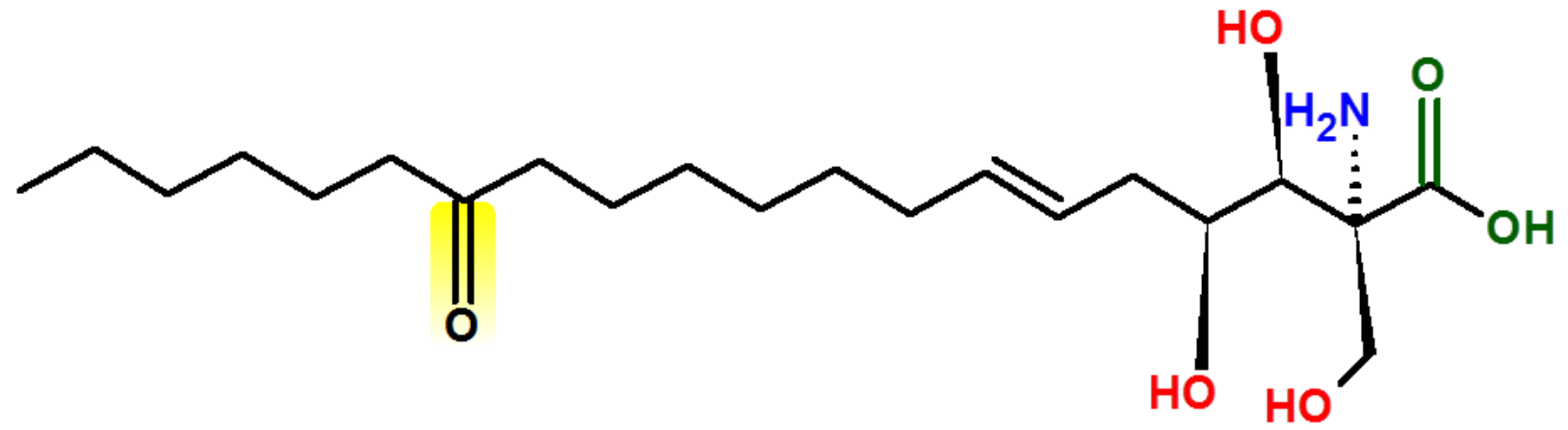

Fig. 1 Molecular formula of Myriocin. Myriocin (MW 401.54) is characterized by conflicting chemical properties (a zwitterionic, multi-functional hydrophilic head and a hydrophobic tail) and displays a limited solubility in water and in common organic solvents 


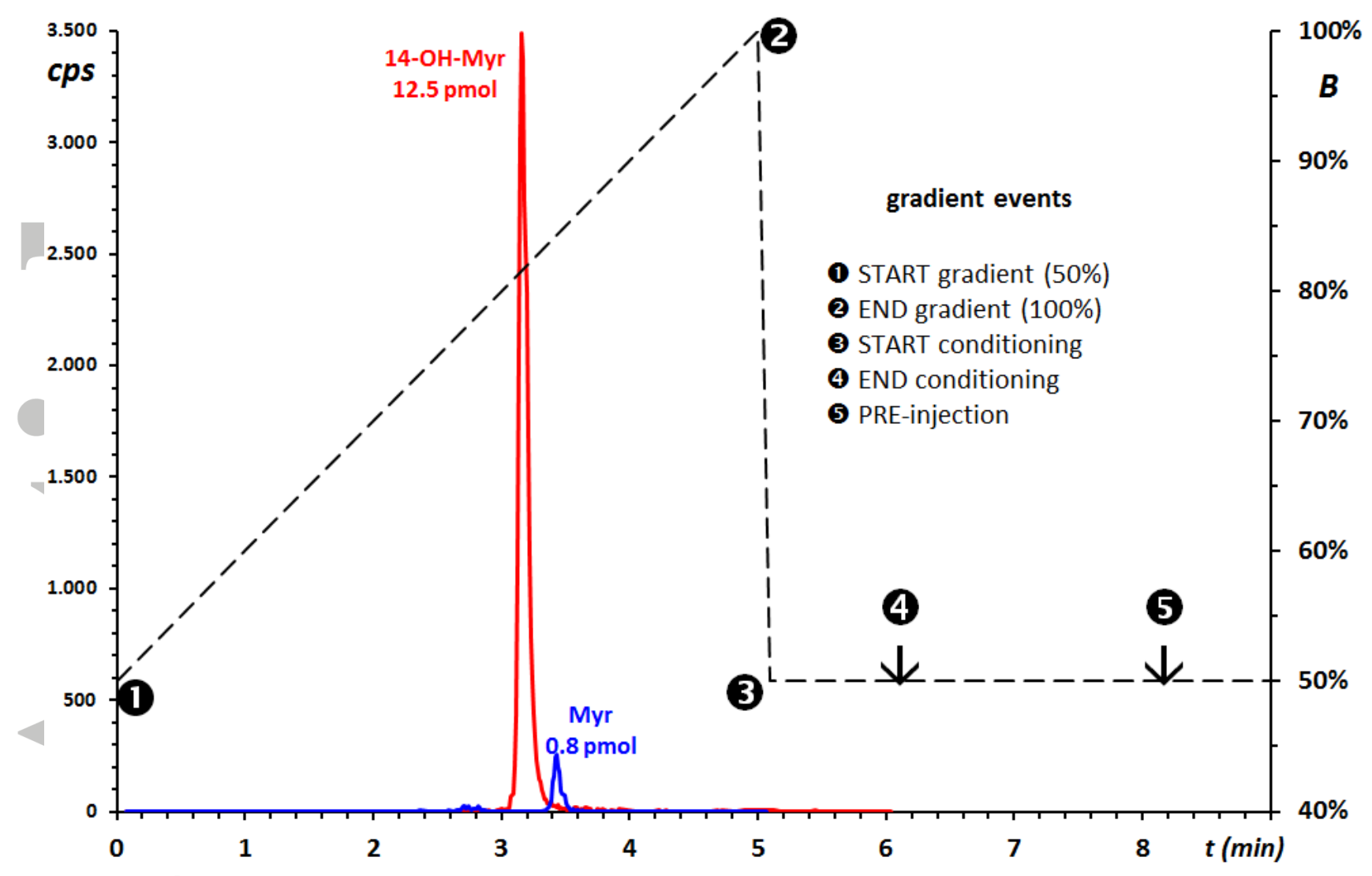

Fig. 2 Chromatogram of Myriocin (Myr), and 14-OH-Myriocin (internal standard, 12.5 pmol) extracted from lung homogenate. A mouse was treated by intra-tracheal nebulization with DMSO-Myriocin (3,15 nmoles), and sacrified $24 \mathrm{~h}$ later. The peaks correspond to a concentration of $0.38 \mathrm{pmol}$ of Myriocin and 12.5 pmoles of 14-OH-Myriocin found in 100 $\mu \mathrm{L}$ of lung homogenate. On-column 25 and 830 fmoles were injected, respectively 

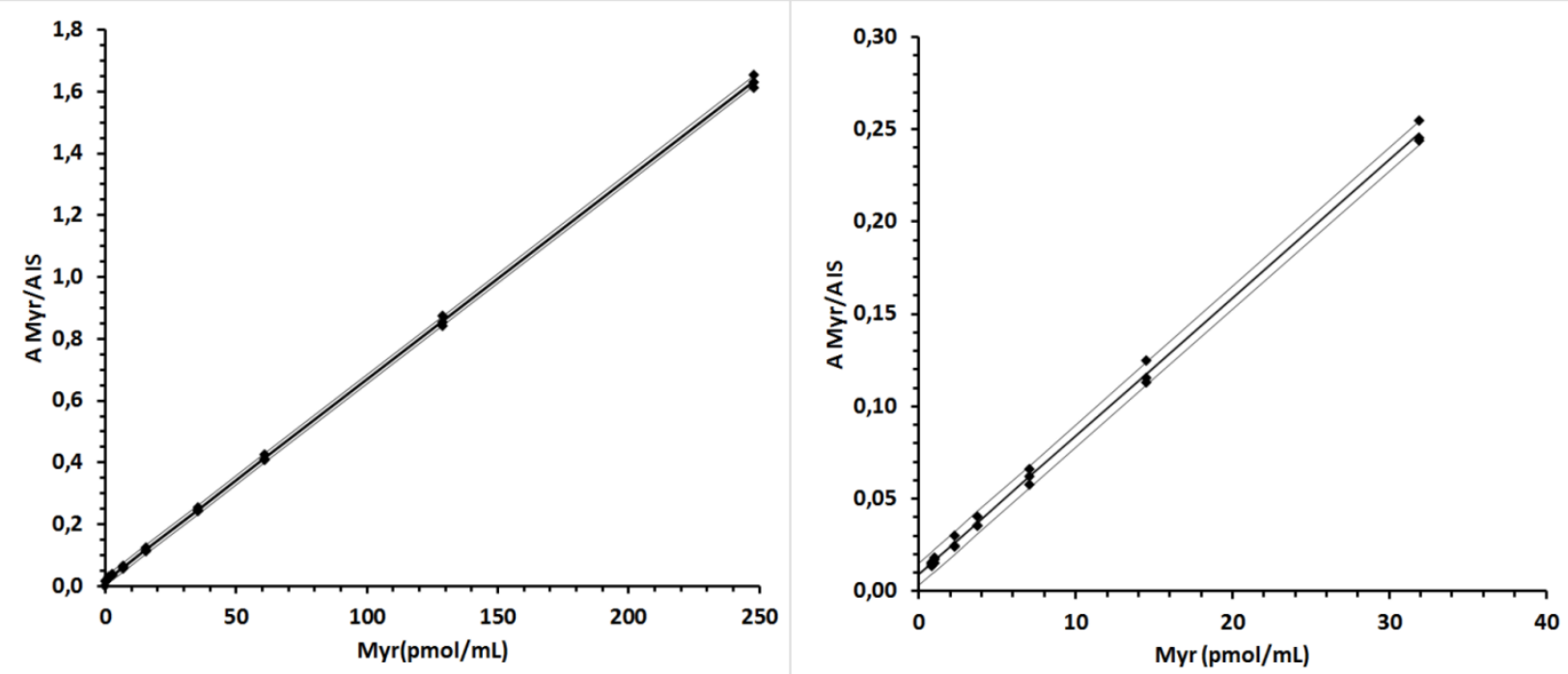

Fig. 3 Examples of calibration curves. The ratio area Myriocin/area IS was plotted against the Myriocin pmoles added to sample. Pure chemical standard Myriocin 0-250 pmoles $/ \mathrm{mL}$ (left panel); Fortified lung homogenate 0-31.2 pmoles/mL (right panel). The points are the 3 actual replicates injected in single analytical run and were prepared as described in Materials and Methods

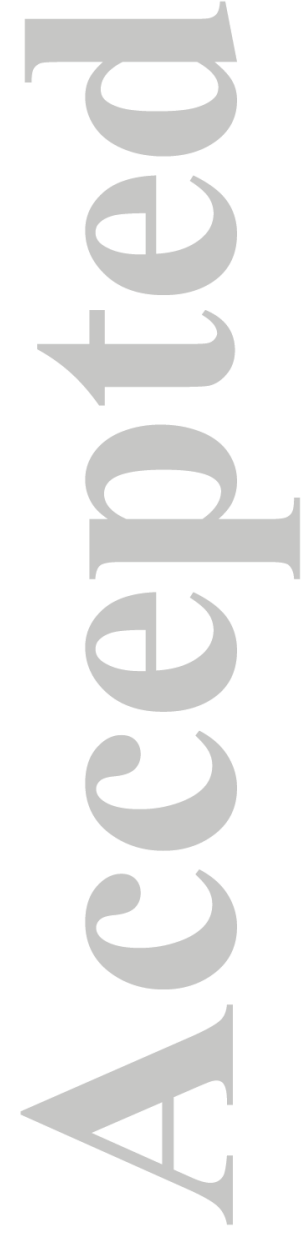




\section{Myriocin}

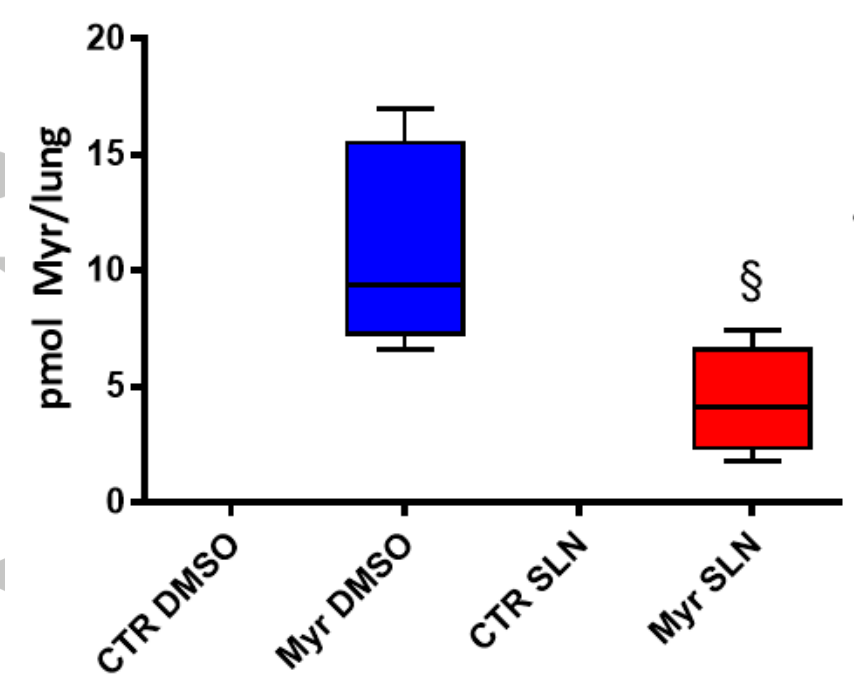

Ceramides

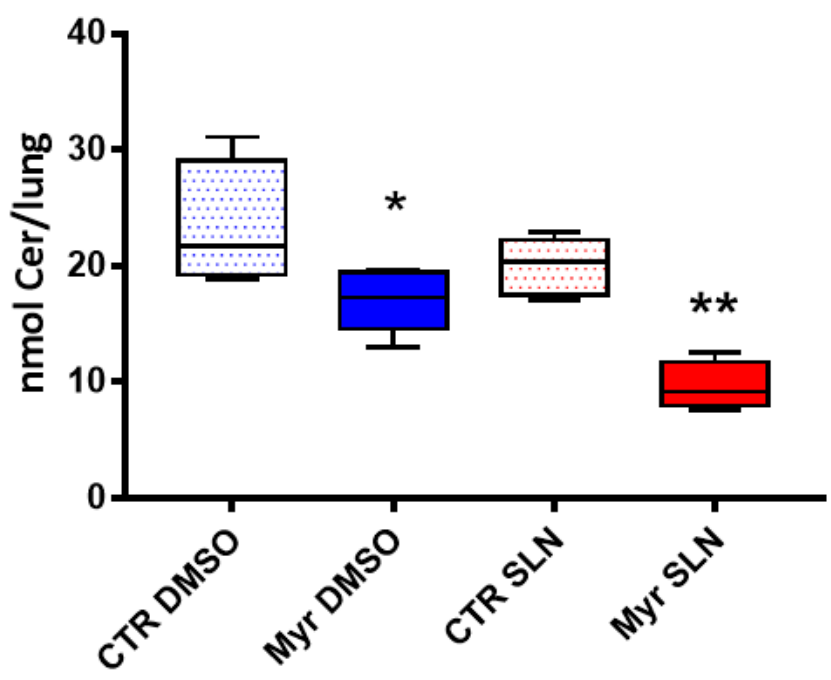

Fig. 4. Myriocin residual content and ceramides concentration after $24 \mathrm{~h}$ from intratracheal administration to mice. Left panel: Myriocin content in mice lung after $24 \mathrm{hrs}$ from DMSO-Myriocin ( $n=6)$ or SLN-Myriocin $(n=4)$ administration (dose 3.15 nmoles and 4.69 nmoles, respectively). After administration of DMSO alone $(n=4)$ or empty SLN ( $n=6)$, Myriocin was undetectable. Right panel: total ceramides concentration in the same lungs used for Myriocin quantification. The box extends from the 25th to 75th percentiles of the distribution, the line plotted in the middle is the median, the whiskers go from the smallest up to the largest value. $* \mathrm{p}<0.05$ vs Control DMSO, $* * * \mathrm{p}<0.001$ vs Control SLN 